\title{
Towards adopting AI techniques for monitoring social media activities
}

\author{
Lina Muhammad Al-Ghamdi* \\ ${ }^{1}$ Department of Information Science, King Abdulaziz University, Saudi Arabia
}

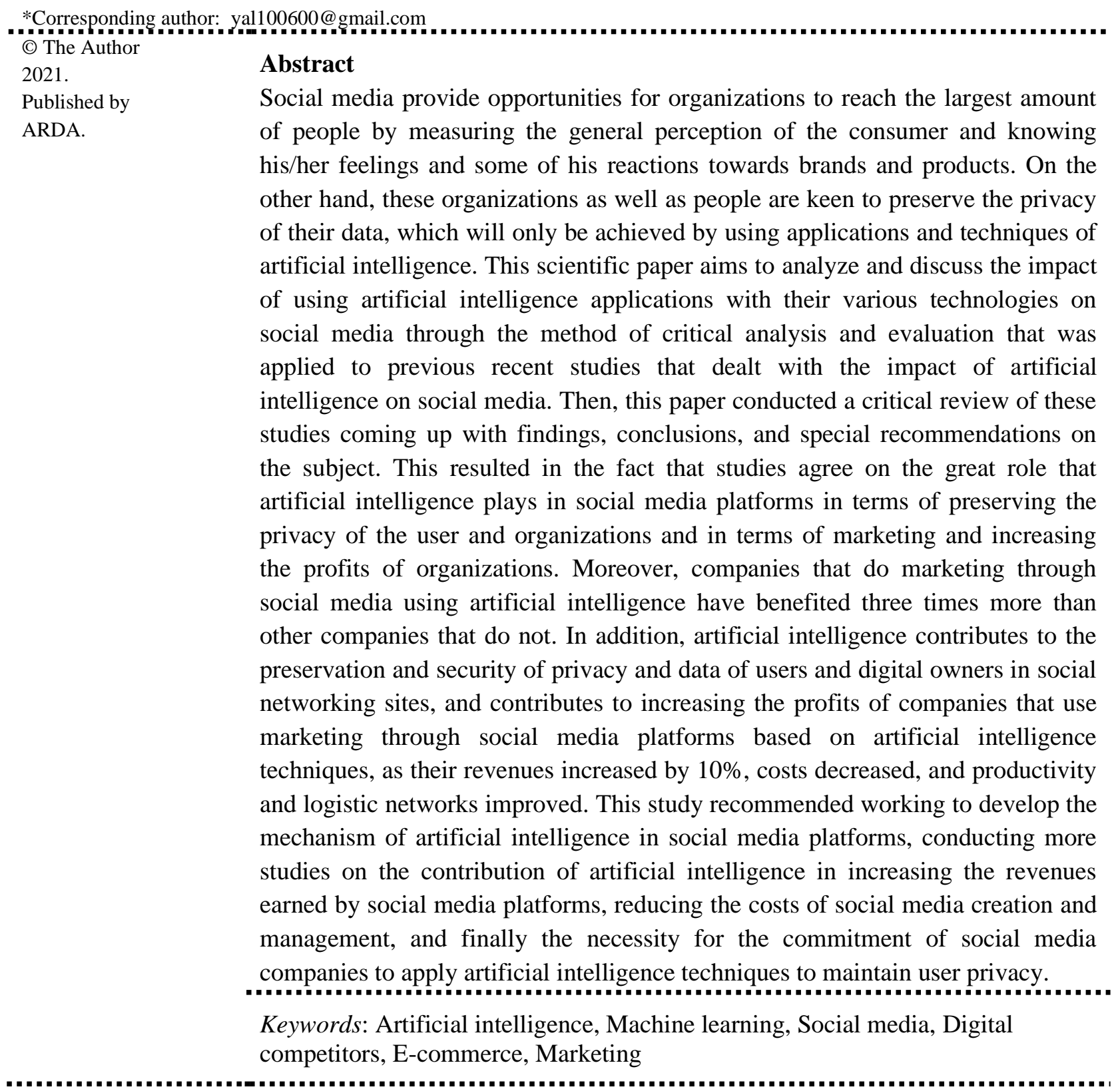

\section{Introduction}

Artificial intelligence is one of the information technologies that affect well-known daily-used informal societies. It uses artificial intelligence outside the camera to control the most prominent personal organizations in the world. For example, Face book uses artificial intelligence techniques to do all aspects of the internet

This work is licensed under a Creative Commons Attribution License (https://creativecommons.org/licenses/by/4.0/ ) that allows others to share and adapt the material for any purpose (even commercially), in any medium with an acknowledgement of the work's authorship and initial publication in this journal. 
user to identify his face in front of the pictures of large target customers by promoting the marketing strategy. Instagram (which is owned by the Face book) also uses artificial intelligence to identify different visuals. LinkedIn also uses artificial intelligence to expand job proposals and advises unemployed individuals or those who have lost their jobs with other job opportunities based on the user's brief. Thus, we find organizations, digital marketers and individuals interact on a daily basis on social media platforms for several social and commercial goals. In fact, the artificial intelligence effectively owns and supervises brand advertising on the Internet and offers unlimited monetary gains to organizations and social media companies [1].

John McCarthy is one of the founders of artificial intelligence after moving to Dartmouth College. The latter became the birthplace of the field of artificial intelligence, at which time McCarthy, Marvin Minsky and Claud Shannon succeeded in bringing together American scientists with an interest in the fields of neural networks, intelligence studies and control theory to participate in a two-month workshop in Dartmouth in the summer of 1956. This workshop resulted in programs and applications such as chess and a thinking program called Logic Theorist by Simon and Newell, where Simon said about it:

"We invented a computer program capable of thinking in non-digital form". However, the main result that continues until present is the adoption of McCarthy's proposal to call the new field at that time "artificial intelligence" to be a stand-alone field adopting a methodology to try to build machines that operate independently in complex and variable environments [2].

This scientific paper will deal with the impact of using artificial intelligence applications on social media through an evaluation critique of a group of recent literature and studies. The importance of this paper is highlighted by the novelty of its studies and its combination between the dependent and independent variables. In addition, it analyzed previous studies that dealt with the topic of artificial intelligence and its impact on Social media and then evaluated these studies to come out with results, conclusions and recommendations that would be beneficial for future researchers in this regard.

This paper contains six sections that introduce the problem of the study and its methodology, then present the previous studies related to the current study and discuss them. in the fifth section with clarifying points of similarities and differences between all studies and finally the results and recommendations that came out of this study.

\subsection{Problem statement}

It has been proven that the use of artificial intelligence applications at every stage of the social mediator (user) helps organizations to display the products they create with promotions in front of customers in a way that cannot be completely clear as much as it is attractive and marketing, in which the current personal organizations benefit by taking advantage of all aspects of social media with the help of artificial intelligence, which operates regularly in the background of the stages that the user pass through [1]. Hence, the main question of this study is what is the reality of using artificial intelligence applications on social media? The following sub questions are derived from it:

- How do organizations and owners benefit from AI applications in social media? - - How do individuals (users) benefit from artificial intelligence techniques in social media?

- What are the future implications of applying artificial intelligence techniques in social media?

\subsection{The research methodology}

This study used the method of clear and transparent critical evaluation and systematic review of published studies. The aim of this method is to analyze and scrutinize these studies and determine their quality, similarities and differences, strengths and weaknesses, and confidence in their results with specific rules and restrictions in order to reach results and recommendations that enhance the subject of the current study and strengthen it. The researcher reviewed journals, periodicals, letters and scientific papers related to the subject of the study. 


\section{Previous studies}

This part of the study reviews recent studies with constructive critical analysis and evaluation. The researcher used magazines, periodicals and scientific letters dealing with the topic of artificial intelligence and its impact on social media arranged chronologically from the most recent to the oldest in the period from 2017 to 2020 .

\section{Study [1] entitled "Artificial Intelligence for social media safety and security"}

This study is based on an evaluation of the role of artificial intelligence in the safety and security of social media and its unique relationship with the usual programming or stages of innovation that help to use artificial intelligence methods to preserve the privacy and security of user data through social media. The importance of the study lies in clarifying the relationship between the independent and the dependent variables where it focused on assessing the role of artificial intelligence in social media in details using the evaluation approach. The most important results of the study were that it clarified the major and fundamental role of artificial intelligence in social media and its ability to quickly identify the danger and thus immediately alert the organization and the user and start the reaction by either expanding or reducing its project, and alert the user to preserve his privacy. The involved organizations and show managers can use artificial intelligence techniques by examining customers' practices and searching for their clients' preferences to reach the intended interests. Artificial intelligence helps organizations create and build great trust with their customers. In addition, it helps supervisors efficiently follow the transformations that are attributed to a superior advertising style, and thus saving a huge amount of money and keeping individuals' safety and security. The study recommended conducting more specialized studies in tackling and examining the role of artificial intelligence methods in social media.

Study [2] This study is similar to the current study with the role of artificial intelligence in social media in terms of monitoring, security and safety that resulted from the great role of artificial intelligence in monitoring social media as in the current study, and the current study benefited from this study as it added a new meaning to the methods of intelligence Artificial Intelligence in preserving the privacy and security of user data in social media, and the current study added many methods of artificial intelligence in protecting social media in various fields.

\section{Study [3] entitled "Social media monitoring: an innovative intelligent approach"}

This study aimed at proposing a social media monitoring platform supported by artificial intelligence with an innovative approach, with the aim of enabling digital marketers to better understand customers with a smart vision. The study focused on monitoring the artificial intelligence methods in social media in the field of digital marketing. The importance of this study lies in its novelty and its attempt to develop an innovative approach in an unprecedented way using the descriptive approach.

Among the most important results of this study is that marketers can take advantage of artificial intelligence methods in monitoring social media to achieve their goals. Artificial intelligence methods can help improve sales and marketing processes, gain a competitive advantage over others and increase revenues. The SIA monitoring platform has been adopted. This is a monitoring and analyzing platform of Social media that helps provide brands with the following services through:

- Listening to what customers say.

- Discovering negative conversations that may tarnish the brand's reputation.

- Spying on competitors to ensure that the brand is on top of the market game.

- Continuously improving social media strategy.

- Obtaining comprehensive advice on how to take action based on ideas.

This study also recommended the necessity of future work on adopting artificial intelligence methods to monitor social media. 
This study is similar to the current study with the role of artificial intelligence in social media in the field of digital marketing, which resulted from the great role of artificial intelligence in improving the buying and selling processes in social media, as in the current study, as this is one of the results of the current study that has benefited the study The current study of this study added a new meaning to the methods of artificial intelligence in digital marketing in social media, and the current study was distinguished by mentioning many methods and methods of artificial intelligence in protecting social media in various fields.

\section{Study [4] entitled "Artificial intelligence ethics: governance through social media"}

The study presents a proposal to facilitate self- machine learning of ethical behavior using a proposed training data that is a mixture of human behavioral data found on social media and related sites, which can help an artificial intelligent machine to make decisions during the process of solving real world problems. The study used the experimental methodology.

The importance of this study is based to its applying of training artificial intelligence systems on ethical performance by sending alerts on social media sites to prevent misuse of these technologies by companies or national governments. The most important results of the study were that the application of an artificial intelligence system trained on social media data on ethical and unethical performance through the process of self-learning can play a new, unrealized role in the governance of future artificial intelligence systems. Moreover, the artificial intelligence system dedicated to human behavior data is exposed to different degrees on the ethical scale and with continuous training it can solve ethical problems in social networking and related sites. The study recommended conducting more studies on the possibility of developing effective mechanisms to allow the artificial intelligence system to keep pace with the latest publications.

This study is similar to the current study with the role of artificial intelligence in social media in terms of allocating it to the role of machine learning, which is considered one of the applications of artificial intelligence in the governance of future artificial intelligence systems and in preserving ethical behavior in social media, and the current study aims to discuss various methods of intelligence Artificial Intelligence in monitoring social media and this study is one of these methods that affect the results of the current study, as it added a new meaning to the methods of artificial intelligence in monitoring social media, and the current study was distinguished by mentioning many methods and methods of artificial intelligence in protecting social media in various fields.

\section{Study [5] entitled "Matching the future capabilities of artificial intelligence-based software for social media} marketing with potential users' expectations"

This study tried to explore the capabilities of future artificial intelligence programs in analyzing marketing through social media and about the audience, images and feelings. It used the descriptive analytical method as it collected data of 150 digital agencies from Romania, Italy and France. The questionnaire was used as a tool with a criterion for determining the different causal configurations of the capabilities of programs based on artificial intelligence. The importance of the study lies in its relying on the best principles that characterized previous approaches adopted by scientists in the systematic literature review. The study problem was the following question: What is the applicability of machine learning technology in marketing through Social media?

The most important results of this study were to prove that the development of a program based on artificial intelligence greatly contributes to marketing through social media. As it used the descriptive and analytical approach, the study applied data collection from three countries, namely Romania, Italy and France with their multiple cultures the results were as following: In Romania, deep learning algorithms have influenced the ability to identify locations and moments of product consumption by analyzing future social media images. In France, the effect of using artificial intelligence methods was to classify each user-generated content and the ability to track the reaction towards new products in the market or promote them through social media by analyzing feelings. Finally, in Italy, the result was the ability to analyze audience through categorizing social 
media with frequent posts from the beneficiary audience. This resulted in the fact that multiculturalism plays an important role in the development of artificial intelligence programs that are reflected in the processing programs used in social media, and that the innovative capabilities of artificial intelligence in media software are required to provide new and continuous solutions to users. It also brings tremendous benefits to researchers' ideas. The study recommended future research to test the impact of AI media and software capabilities on improving the work of social digital agencies for media marketers.

This study is similar to the current study with the role of artificial intelligence in social media in terms of analyzing the audience, feelings and images that affect the monitoring of marketing through social media, and the current study aims to discuss various methods of artificial intelligence in monitoring social media and this study is considered one Among these methods that affect the results of the current study, as it added a new meaning to the methods of artificial intelligence in monitoring social media, and the current study was distinguished by mentioning many methods and methods of artificial intelligence in protecting social media in various fields.

\section{Study [6] entitled "Exploring artificial intelligence techniques applicability in social media marketing"}

The main objective of this study is both to examine the correlations between experience in social media marketing and the level of knowledge regarding the applicability of ML artificial intelligence techniques in SMM (Social Media Marketing) and the frequency of ML algorithms use in SMM campaigns. The study used the evaluative and descriptive approach that contributed to highlight the importance of the study since it is a result of the Future Web project and it focused on analyzing three very important groups namely audience analysis, image analysis, and feelings analysis.

The study result was that marketing experts' learning through social media of how to adopt artificial intelligence technologies will greatly benefit them and their related companies or projects. It recommended that decision-makers should encourage investments in technologies such as future artificial intelligence media programs in a way that leads to more long-term profits beside the short-term gains.

This study is similar to the current one in that it deals with the relationship of experience in the field of marketing through social media and the level of knowledge regarding the possibility of applying artificial intelligence techniques in audience analysis, sentiment analysis and image analysis that affects monitoring marketing through social media, and the current study aims to discuss various Artificial intelligence methods in monitoring social media, and this study is one of these methods that affect the results of the current study, as it added a new meaning to the methods of artificial intelligence in monitoring social media, and the current study was distinguished by mentioning many methods and methods of artificial intelligence in protecting social media. In various fields

\section{Study [7] entitled "Machine learning and social media in crisis management: agility vs. ethics"}

This study aims at analyzing the ethical risks and the implications of using an automated system that learns from social media data to provide intelligence information in crisis management using the experimental approach. The problem of the study is the following question: How do artificial intelligence systems limit the risk of data in Social Media? The importance of this study lies in its proposed scenario as an experimental example on which it is based. Then, it comes out with realistic practical results and recommendations that have proven successful. Among these results is an algorithm in machine learning (ML) that helps in following an ethical approach in social media that alerts the user to the presence of danger and helps to fix or avoid it, the development of a new generation of machine learning-based social media analysis tools to support emergency situations. The study also recommended making more efforts and realistic experiments in relation to the role of artificial intelligence in social media during emergencies and the risks of data privacy.

This study is similar to the current with an analysis of moral hazards and the implications of using artificial intelligence techniques to provide intelligence information in crisis management through social media, and the current study aims to discuss various methods of artificial intelligence in monitoring social media, and this 
study is one of those. The methods that affected the results of the current study, as it added a new meaning to the methods of artificial intelligence in monitoring social media sites, and the current study was characterized by mentioning many methods and methods of artificial intelligence in protecting social media in various fields.

Study [8] entitled "Using artificial intelligence on social media's user generated content for disruptive marketing strategies in ecommerce"

This study aims at examining and analyzing digital marketing methods appropriate for e-commerce by collecting images of a brand by the consumer through social media sharing via artificial intelligence, and how brands communicate with the target audience using the descriptive analytical approach. The importance of the study lies in its widespread and important topic in the current era of collecting and sharing photos on social media.

The results of this study are that images are the preferred method for expressing the popularity of brands on social media platforms, and that understanding the information that users share and applying this knowledge in marketing strategies can represent a great competitive advantage. Artificial intelligence contributes to targeting the desired audience by understanding how collecting keywords and images that users share through social media, which helps marketing managers to create marketing campaigns for the product through the elements that usually appear in the pictures with the product. Presenting the product in e-commerce has an impact on the user's purchase intentions using artificial intelligence techniques. The study recommended the need to conduct more future studies on the advantages of applying artificial intelligence techniques in the field of digital marketing on social media.

This study is similar to the current study in that it aimed to examine and analyze appropriate digital marketing methods for e-commerce by collecting images of a brand by the consumer through social media, and this is done by means of artificial intelligence techniques, and this is what the current study seeks to discuss, as it aims to discuss various methods Artificial Intelligence in monitoring social media, and this study adds to the current study a new method and method that supports the results of the current study, as it added a new meaning to artificial intelligence methods in monitoring social media sites, and the current study was distinguished by mentioning many methods and methods of artificial intelligence in protecting social media in various fields should be clear and concise.

\section{Results and discussion}

Similar to the findings of previous studies, the current study has shown that, the role of artificial intelligence in social media in the field of digital marketing resulted that $\mathrm{AI}$ is improving the buying and selling processes in different aspects. it also added a new meaning to the methods of AI and was distinguished by mentioning many methods of how AI can protect the social media in various fields. We noted from previous studies that there are similarities and differences between a group of studies that have been analyzed in this paper. In this part, we will deal with these similarities and differences and discuss the findings of previous studies in some detail, in addition to critically evaluate them in terms of their strengths and shortcomings from the researcher's point of view as follows:

All studies agreed on the great and effective role of applying artificial intelligence techniques in social media, but they differed in the nature of the impact that results from the use of artificial intelligence applications in social media. The views of each study varied according to the field that they addressed, so we find similarities between studies [1], [4] and [7] as they focused on the safety and security of social media, the privacy of user data, and the privacy of organizations and owners' data in terms of the speed of knowing the danger. This means the speed of alerting the individual or organization, providing intelligence information, and the contribution of artificial intelligence to reduce the threat of data privacy by training and using ML technology. Study [1] was characterized by its handling of various topics related to artificial intelligence and its role in the safety and security of social media. However, it lacked further studies to support its theories. Study [4] was characterized by the application of the experimental approach and the adoption of a successful experiment in studying an artificial intelligence system and knowledge of its ability in facing the ethical challenges of human behavior in social media and related sites. Study [7] was distinguished by presenting many realistic examples that occurred recently in real life, and how artificial intelligence systems were able to contribute to reducing the risk of earthquakes, terrorism, and more. 
The above studies differed from study [3], which focused on exploring the role of the artificial intelligence field for digital marketers in terms of understanding consumer desires, working on effective marketing and achieving profitability goals by taking advantage of artificial intelligence. This agreed with both studies [5] and [6]. Study [5] focused on the field of digital marketing through social media, exploring the capabilities of artificial intelligence, images, audience and feelings, and then working on digital marketing for their products, thus increasing the financial profit by using ML technology, which is considered one of the most important techniques of artificial intelligence. These studies are similar to study [8] that examined and analyzed digital marketing on social media and e-commerce with the aim of increasing sales leading to financial profit for organizations and owners and brands reaching the target audience on social media platforms. Study [3] was distinguished by the abundance of graphical and statistical analyzes that support the results of the study, but it lacked to enrich the theoretical part. As for study [5], it was distinguished by many supporting evidence. It dealt with previous studies related to analysis, discussion and the use of the analysis of the materials that made up the study, namely pictures, the public, and feelings. Study [6] was also distinguished by mentioning many previous studies that support its topic and the statistical tables that prove the validity of its theories. Finally, study [8] was characterized by a theoretical part that is rich in definitions and information covering the subject of its study and the frequent citation of previous studies as a supporting background to the topic. With reference to the statistics of previous studies, we find that the use of artificial intelligence techniques in marketing through social media platforms and related sites had a significant impact on organizations in increasing sales and profits resulting from marketing through social media, as shown in Fig.1.

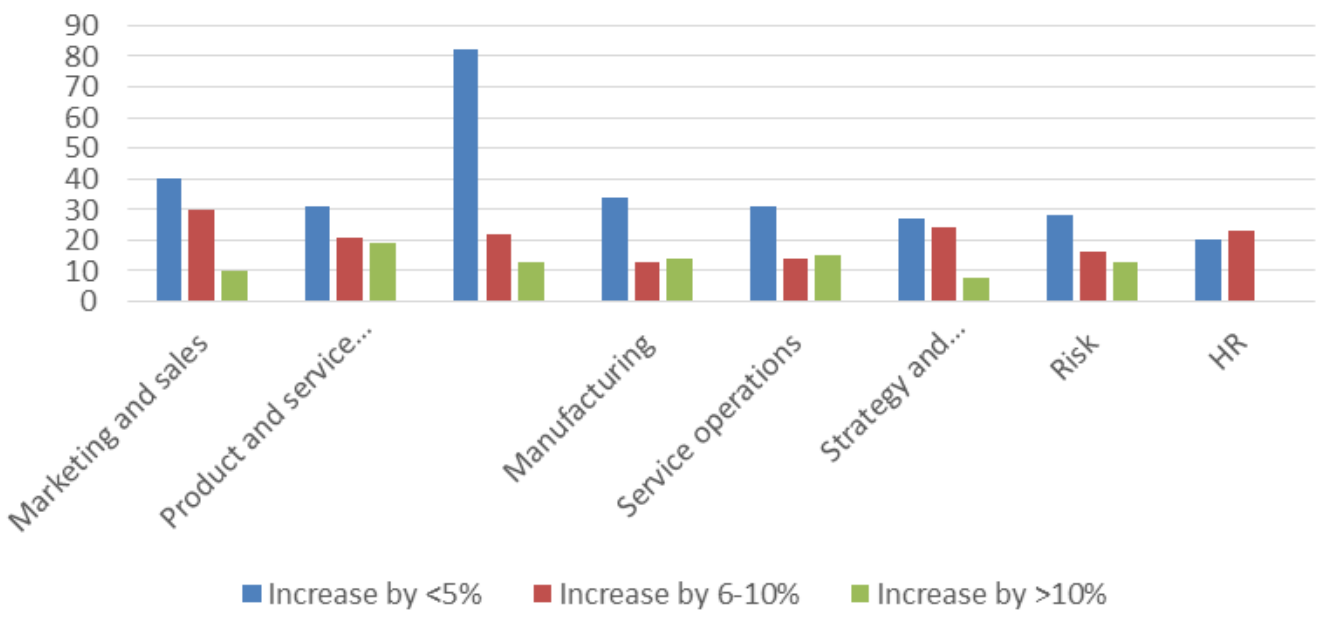

Figure 1. Average increase in revenue [9]

Respondents reported that the revenue growth from AI use cases in marketing and sales, product and services are dramatically increased by almost $\sim 40-80 \%$. Considering the other domains affected, more than $6-10 \%$ increased HR and service operation. Mckinsey Company conducted a study on the impact of artificial intelligence in marketing through social media platforms and related sites. It found that $63 \%$ of respondents confirmed that the companies that used artificial intelligence techniques were three times better than other companies were, and that revenues exceeded $10 \%$ due to applications of artificial intelligence in marketing on Social platforms as indicated by the statistics above.

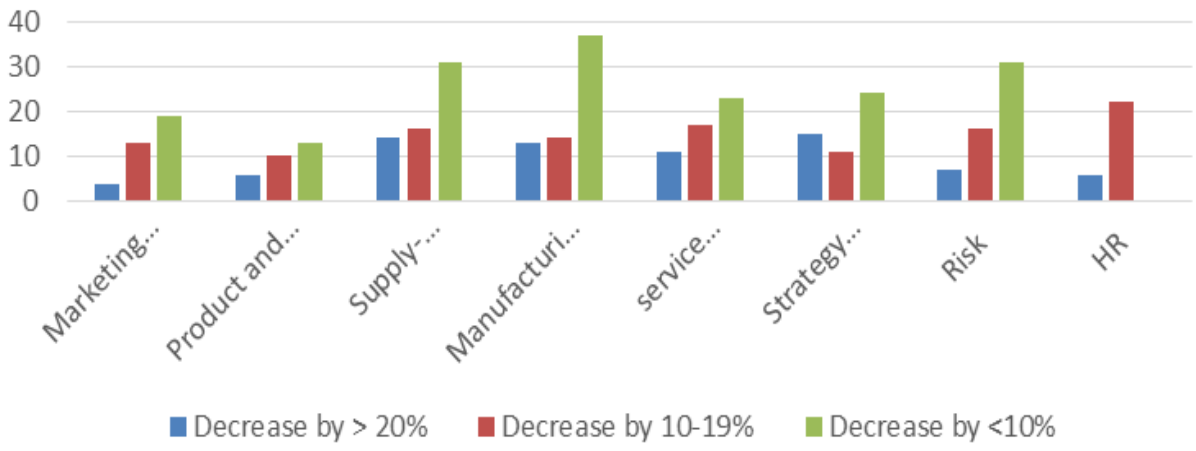

Figure 2. Average cost reduction [9] 


\section{Conclusion and recommendations}

Through analyzing previous studies and referring to books and scientific studies, we summarize the most important conclusions that came out of the current study as follows:

- There is a huge impact of the use of artificial intelligence techniques on social media.

- Companies that marketed through social media using artificial intelligence have benefited three times more than other companies have.

- Artificial intelligence contributes to the preservation and security of privacy and data of users and digital owners in social networking sites.

- The use of artificial intelligence contributes to increasing the profits of companies that use marketing through social media platforms based on artificial intelligence techniques.

This study recommends the following:

- The need to work on developing the artificial intelligence mechanism in social media platforms.

- The imperative to conduct more studies on the contribution of artificial intelligence to increasing the revenues generated by social media platforms, and reducing the costs of creating and managing social media.

- The necessity of conducting experiments and statistics for the uses of artificial intelligence and its impact on the user and the marketer on social media.

- The necessity of the commitment of social media companies to apply artificial intelligence techniques to maintain user privacy for them.

\subsection{References}

[1] S. Malik, "Artificial Intelligence for Social Media safety and security," International Engineering Journal, vol.5, pp. 5-7, 2020.

[2] Alratemi. Mohammed Ali, "Artificial Intelligence and Expert Systems," Libyan Academy of Graduate Studies. First edition. Eygpt, 2012.

[3] E. Perakakis. and G. Mastorakis, "Social Media Monitoring: An Innovative Intelligent Approach," Mdpi journal designs, vol.3, no.2, pp. 1-12, 2019.

[4] B. Jorge and A. Ross, "Artificial Intelligence Ethics: Governance through Social Media," Conference Paper, IEEE International Symposium on Technologies for Homeland Security.vol.10, no. 1109, November, pp 1-4, 2019.

[5] C. Alexandru and K. Maher "Matching the future capabilities of an artificial intelligence-based software for social media marketing with potential users' expectations," Technological Forecasting \& Social Change, vol.151, no. 119794, pp 10-15, 2019.

[6] E. Angela.and C.Alexandru "Exploring Artificial Intelligence Techniques' Applicability in Social Media Marketing," Journal of Emerging Trends in Marketing and Management, vol 1, no. 1, September, pp. 156-164, 2018.

[7] G. Marius and E. Angela, "Using Artificial Intelligence on Social Media's User Generated Content for Disruptive Marketing Strategies in ecommerce." Annals of "Dunarea de Jos" University of Galati Fascicle I. Economics and Applied Informatics, vol.10, no. 3, December, pp.5-11, 2018.

[8] L. Vitaveska, "Machine Learning and Social Media in Crisis Management: Agility vs. Ethics," Proceedings of the 14th ISCRAM Conference, vol.13, no. May, pp.256-260, 2017. 